\title{
Integrating Immunotherapy Into the Management of Renal Cell Carcinoma
}

\author{
Matthew Zibelman, MD, and Elizabeth R. Plimack, MD, MS
}

\begin{abstract}
Before 2005, systemic treatment of metastatic renal cell carcinoma (RCC) was limited to a few minimally effective options. Since then, new agents have emerged targeting the vascular endothelial growth factor and mTOR pathways, which has improved outcomes for patients. Options increased even further beginning in 2015 with 3 new agents, including the addition of nivolumab, the first immune checkpoint inhibitor to demonstrate improved survival in RCC. RCC has long been considered a malignancy with immunogenic potential, and nivolumab offers the potential for durable responses in some patients with a generally tolerable toxicity profile. With so many drugs available to clinicians and patients, properly integrating immune checkpoint blockade (ICB) into the treatment paradigm is challenging. Additionally, emerging research with other ICB agents, as well as ongoing trials of combination strategies, is likely to further impact clinical decisionmaking. This article attempts to provide some context to inform systemic treatment decisions in the current landscape, with a particular emphasis on the role of immunotherapy, outlines the ongoing immunotherapy research in RCC, and discusses how treatment may evolve.

J Natl Compr Canc Netw 2017;15(6):841-847 doi:10.6004/jnccn.2017.0103
\end{abstract}

The emergence of immunotherapy heralded by drugs targeting immune checkpoints has generated tremendous promise across the oncology community and dramatically altered the therapeutic landscape of many malignancies. Management of renal cell carcinoma (RCC), a cancer long considered "immunogenic," is a prominent example. In the 1960s, cases of spontaneous remissions in patients with metastatic RCC (mRCC) were reported, documenting a curious phenomenon unique to few metastatic tumors and suggesting an innate ability of the immune system to achieve durable cancer control. ${ }^{1-3}$ In the 1980s and 1990s, interleukin-2 (IL-2) demonstrated the capacity to induce durable remissions in $5 \%$ to $7 \%$ of patients, albeit with significant toxicity, sparking hope for the possibility of a "cure" in patients considered incurable. ${ }^{4,5}$ Now, immune checkpoint blockade (ICB), with drugs targeting the programmed death 1 (PD-1) pathway, has revolutionized the treatment paradigm and laid the groundwork for further innovation in immunotherapy. Despite this potential,

From Department of Hematology/Oncology, Fox Chase Cancer Center, and Temple Health, Philadelphia, Pennsylvania.

Dr. Zibelman receives institutionally directed research funding from

Horizon Pharma and served on an advisory board for EMD Serono. Dr.

Plimack receives institutionally directed research funding from Merck most patients do not respond to ICB, and no clear biomarkers exist to identify patients most likely to benefit. Thus, strategies to increase response rates and optimize outcomes for patients are needed. This may be achieved in a wide variety of ways, including but not limited to drugs with novel mechanisms, rational combination approaches, and using ICB earlier in the disease trajectory. Fortunately for patients with mRCC, in addition to these new immuno-oncology drugs, novel targeted therapies such as cabozantinib and lenvatinib have recently been approved in the metastatic space. Additionally, the adjuvant space has seen its first positive study, with sunitinib meeting its primary end point of diseasefree survival (DFS) versus placebo in patients after curative-intent nephrectomy, but unfortunately with no discernable effect on overall survival (OS) to date. ${ }^{6}$ Without clear evidence-based data from randomized trials to guide practice among the available agents, it can be challenging to decide how best to sequence agents to provide maximal benefit to patients. The Society for and Bristol-Myers Squibb, and has served as a consultant for Bristol-Myers Squibb, Pfizer, Novartis, Genentech/Roche, and Eli Lilly.

Correspondence: Elizabeth R. Plimack, MD, MS, Fox Chase Cancer Center, Department of Hematology/Oncology, 333 Cottman Avenue, Philadelphia, PA 19111-2497. E-mail: elizabeth.plimack@fccc.edu 
Immunotherapy of Cancer (SITC) has published a consensus statement from a convened task force of experts in RCC hoping to shed some light on some of the issues surrounding available immuno-oncology drugs. ${ }^{7}$ But this resource currently relies on expert opinion due to the lack of hard evidence. RCC in many ways now suffers from an embarrassment of riches-there are many drugs to choose from, but little guidance on how best to use them. In this article, we attempt to provide some context to inform decisions on how to integrate immunotherapy into the current treatment landscape for RCC based on currently available data, and to hypothesize as to how these new paradigms may evolve.

\section{Treatment of Patients With mRCC}

\section{Second-Line and Beyond}

Nivolumab, a monoclonal antibody (mAb) targeting PD-1, became the first checkpoint inhibitor to earn FDA approval for treatment of patients with mRCC who had progressed after prior treatment with a tyrosine kinase inhibitor (TKI) by demonstrating improved OS versus everolimus in a phase III trial (CheckMate 025). ${ }^{8}$ Although the overall response rate (ORR) for nivolumab-treated patients in this study was a modest $25 \%,>30 \%$ of these responders achieved durable benefit lasting $>12$ months. This durability of benefit in responders, the so-called "tail" of the survival curve, underscores the true value of nivolumab, and is graphically more evident in an updated survival curve presented at the 2016 International Kidney Cancer Symposium, which reported $29 \%$ of nivolumab responders retained response with a median follow-up of 26 months. ${ }^{9}$ At the time of its approval, second-line options outside of a clinical trial for most patients consisted of either a TKI targeting vascular endothelial growth factor (VEGF) receptor (either axitinib, sorafenib, or TKI of choice not used in the first line between sunitinib/ pazopanib) or an mTOR inhibitor (everolimus or temsirolimus). A third mechanistic option, interferon alfa plus bevacizumab, was rarely used. ${ }^{10}$ Due to its durability of benefit, nivolumab became the de facto second-line option for most patients with mRCC after progression on a TKI.

Since the approval of nivolumab in TKI-refractory mRCC, both lenvatinib (in combination with everolimus) and cabozantinib have achieved FDA approval based on randomized data demonstrating improved progression-free survival (PFS), and in the case of cabozantinib, improved OS as well. ${ }^{11-13}$ The availability of these 3 new agents provides new options for patients, but all were tested in the secondline (or beyond) space, and no level 1 data exist comparing them with each other. Within the limitations of cross-trial comparison, one can infer some clues from the available data. All of these contemporary studies tested their drug against a common comparator-everolimus. Both nivolumab and cabozantinib demonstrated an improvement in OS beyond the first-line setting as of December 2016, whereas lenvatinib has exhibited a PFS benefit. ${ }^{8,12,13}$ However, the performance of the control everolimus arm differed somewhat across the studies, likely reflecting subtle differences in the populations. PFS for everolimus was 4.4 months in the CheckMate 025 study, ${ }^{8}$ 3.8 months in the METEOR study ${ }^{13}$ with cabozantinib, and a high of 5.5 months in the lenvatinib/ everolimus study. ${ }^{12}$ Patients in the lenvatinib study could only have received one prior VEGF-targeted therapy (thus a pure second-line study), which may have contributed to the improved responses because this was a healthier population. Patients in the other 2 studies may have been second or third line, or beyond in the cabozantinib study, which included $>25 \%$ of patients third line or later, whereas patients in the CheckMate 025 trial could not have been beyond third line. The METEOR trial with cabozantinib also allowed treated brain metastases. Thus, the differing efficacy rates reflect the make-up of the included populations. Data supporting the approval of nivolumab and cabozantinib originate from randomized phase III trials, each with $>650$ patients, whereas the approval of lenvatinib stems from a randomized phase II study of 153 patients over 3 arms (the combination and each drug alone). Nivolumab was overall well-tolerated, with grade $\geq 3$ treatmentrelated adverse events (TRAEs) occurring in 19\% of patients. Notably, $68 \%$ of patients receiving cabozantinib experienced TRAEs grade $\geq 3$. There was one grade $5 \mathrm{AE}$ attributed to cabozantinib, with no AEs attributed to nivolumab, and $60 \%$ of patients treated with cabozantinib required a dose reduction. In the combination arm of the lenvatinib/everolimus study, $71 \%$ of patients experienced a grade $3 / 4$ TRAE and $24 \%$ discontinued treatment due to toxicity. Given the totality of these data, nivolumab appears to be the 
preferred option for treatment for most patients with TKI-refractory mRCC (without a contraindication) given its documented OS benefit, potential of a durable response, and favorable toxicity profile.

Still, most patients do not benefit from receiving single-agent nivolumab. Thus, ongoing research is geared toward increasing clinical benefit, with 3 viable options to improve patient outcomes: a better immuno-oncology alternative, a suitable combination approach, or a reliable predictive biomarker to identify who is most likely to benefit (or not benefit). Several other PD-1 pathway inhibitors are being explored in TKI-refractory mRCC. Atezolizumab, a $\mathrm{mAb}$ targeting the primary ligand for PD-1 (PD-L1) often found on the tumor cell, was evaluated in a phase IA basket trial including patients with mRCC with both clear cell RCC (ccRCC) and non-clear cell RCC (nccRCC) ${ }^{14}$ In patients with ccRCC, the ORR was $15 \%$, with median OS (28.9 months) and toxicity data favorably comparable with nivolumab.

\section{First-Line Metastatic}

Current standard of care for first-line treatment of most patients with mRCC is either sunitinib or pazopanib, but for a subset of patients, immunotherapy using high-dose IL-2 (HD IL-2) remains a consideration. In the 2016 consensus statement by the SITC RCC task force, there was a lack of consensus regarding the role of HD IL-2 since the emergence of TKIs and ICB, although two-thirds of members still felt the option should be discussed with all appropriate patients. ${ }^{7} \mathrm{HD}$ IL-2 has demonstrated the capacity to provide long-term remissions in a small subset of patients, with approximately $7 \%$ attaining a complete response, and approximately 5\% alive and diseasefree after 65 months. ${ }^{4,5,15}$ Despite this promise, HD IL-2 administration requires in-patient admission and is associated with marked toxicity appropriate only for patients with optimal clinical and physiologic features. ${ }^{7}$ Consequently, HD IL-2 has become increasingly marginalized and is only available to a small subset of healthy patients with ccRCC and access to treatment at high-volume centers.

ICB (generally in combination therapy) as a first-line option is being evaluated in several randomized trials and may further alter the treatment paradigm, affecting downstream second-line options. Efficacy data on single-agent nivolumab as firstline therapy for mRCC is limited, with the largest published cohort consisting of 24 patients without prior systemic therapy. ${ }^{16}$ This group received nivolumab at $10 \mathrm{mg} / \mathrm{kg}$, resulting in 2 complete responses, an ORR of $13 \%$, and a 48-week PFS rate of $39 \%$. These data from a small patient cohort would require further validation, but challenge the longaccepted paradigm that response rates are higher in earlier lines of therapy, and suggest that nivolumab may work better after prior treatment with a TKI, perhaps due to changes to vasculature or tumor antigens occurring in response to VEGF inhibition. Phase III trials of single-agent ICB are not being pursued in the first-line setting.

Combination approaches with ICB are in various stages of clinical testing and are promising potential techniques to increase the percentage of patients who clinically benefit. These approaches are varied and include combined ICB, ICB with a VEGF-targeting TKI, and combining ICB with cytokines or other agents geared to favorably enhance the immune milieu. The goal is to increase efficacy without compromising safety, which so far has proven challenging. Dual checkpoint blockade (ie, adding an agent targeting a separate checkpoint in addition to PD-1) has already garnered approval for improved survival in the first-line treatment of metastatic melanoma using the combination of nivolumab with the cytotoxic T-lymphocyte-associated protein 4 (CTLA-4)-specific mAb ipilimumab. This combination demonstrated improved efficacy over both single agents, but with a consequent increase in grade 3/4 TRAEs. ${ }^{14,15}$ This same combination was initially evaluated in $\mathrm{mRCC}$ as part of the CheckMate 016 trial, a multiarm phase I study assessing varying combination doses of nivolumab and ipilimumab for patients with both TKI-refractory and untreated mRCC, with updates last reported at the 2016 ESMO Annual Meeting. ${ }^{16}$ Combined data across 2 dose combinations demonstrated an ORR of approximately $40 \%$ and a disease control rate close to $80 \%$ (complete and partial responses plus stable disease). However, grade 3/4 TRAEs occurred in $38 \%$ and $61 \%$ of patients, respectively, across the 2 dose combinations reported (nivolum$\mathrm{ab}, 3 \mathrm{mg} / \mathrm{kg}+$ ipilimumab, $1 \mathrm{mg} / \mathrm{kg}$, or the opposite). The same CheckMate 016 study also included arms combining nivolumab with sunitinib or pazopanib. Due to increased hepatotoxicity on the TKI arms, both arms were ultimately halted for further study, 
dampening enthusiasm for combination PD-1/TKI approaches. ${ }^{17}$ However, hope has been renewed with the presentation at ESMO 2016 of the combination of the PD-1 inhibitor pembrolizumab with axitinib, a multitarget TKI approved for treatment of patients with $\mathrm{mRCC}$ who have progressed on a prior TKI. Preliminary results showed the combination to be well-tolerated, with grade $\geq 3$ immune-related AEs occurring in $19.2 \%$ of patients and without significant increases in diarrhea or hepatotoxicity. ${ }^{18}$ The ORR was an impressive $71.2 \%$, with a disease control rate of $90.4 \%$. Evidence from combinations of ICB with other therapeutic strategies remains too immature for current discussion.

Because of the impressive efficacy of dual ICB with nivolumab and ipilimumab reported in both the first- and second-line settings, albeit with increased toxicity, a randomized phase III trial of this combination versus sunitinib in previously untreated mRCC patients completed accrual (CheckMate 214; ClinicalTrials.gov identifier: NCT02231749), with results pending. ${ }^{17}$ Similarly, based on data presented on axitinib and pembrolizumab, an ongoing phase III trial randomizing this combination against standard sunitinib is currently accruing patients (KEYNOTE-426; ClinicalTrials.gov identifier: NCT02853331). Several other first-line combination trials are also ongoing or planned (Table 1). As a result, although TKIs remain the standard for first-line treatment of mRCC outside of a clinical trial, it is highly likely that combination ICB approaches will achieve regulatory approval within the next few years. The difficulty in choosing optimal therapy may prove to be similar to that for TKIrefractory disease, namely that multiple combinations could prove to be superior to sunitinib, but data comparing the combinations directly will be lacking, making it challenging for clinicians to differentiate.

\section{(Neo)Adjuvant}

Clearly, the role of immunotherapy for patients with $\mathrm{mRCC}$ is rapidly evolving, thus the next frontier appears to be determining whether there is a place for ICB in patients with localized disease. The Sunitinib Treatment of Renal Adjuvant Cancer (S-TRAC) met its primary end point of improving DFS, but without a significant OS benefit to this point; on the heels of the negative results of the adjuvant ASSURE trial (Adjuvant Sorafenib and Sunitinib for Unfavorable Renal Carcinoma), no definitive adjuvant therapy option yet exists. ${ }^{6,18}$ Although we await updated survival analysis from S-TRAC and other unreported adjuvant TKI studies, ICB has entered the clinic in several trials in the adjuvant or neoadjuvant setting. The promise and potential upside is clear: eradicating microscopic residual disease to increase the cure rate after curative-intent surgery. Single-agent PD-1 pathway-targeting drugs have repeatedly been shown to be well-tolerated by most patients, thus it is reasonable to offer them before and after surgery. But as these trials begin to enroll patients, several important issues regarding trial design, clinical rationale, and feasibility are worth considering.

First, whether to administer ICB agents preoperatively, postoperatively, or both in the early disease setting is unclear and controversial. A purely adjuvant study is clean and simple, with patients with high-risk disease recruited after recovery from surgery. This allows for a clear workflow of patients be-

\begin{tabular}{|c|c|c|c|}
\hline ClinicalTrials.gov Identifier & $\begin{array}{l}\text { Combination } \\
\text { Arms }\end{array}$ & $\begin{array}{l}\text { Control } \\
\text { Arm }\end{array}$ & Primary Outcome \\
\hline $\begin{array}{l}\text { NCT02684006 } \\
\text { (JAVELIN Renal 101) }\end{array}$ & Avelumab + axitinib & Sunitinib & Progression-free survival \\
\hline NCT02420821 & Atezolizumab + bevacizumab & Sunitinib & $\begin{array}{l}\text { Progression-free survival } \\
\text { and } \\
\text { Overall survival (PD-L1-positive } \\
\text { patients ONLY) }\end{array}$ \\
\hline $\begin{array}{l}\text { NCT02853331 } \\
\text { (KEYNOTE-426) }\end{array}$ & Pembrolizumab + axitinib & Sunitinib & $\begin{array}{l}\text { Progression-free survival } \\
\text { and } \\
\text { Overall survival }\end{array}$ \\
\hline NCT02811861 & $\begin{array}{l}\text { Pembrolizumab + lenvatinib } \\
\text { OR } \\
\text { Lenvatinib + everolimus }\end{array}$ & Sunitinib & Progression-free survival \\
\hline
\end{tabular}


ing referred after surgery by urologists to medical oncologists, removing the potential impact of systemic therapy on surgical timing and outcome, and minimizing surgical resistance to referring for preoperative treatments that could delay surgery. However, the efficacy of ICB may prove less relevant in the adjuvant space. Mechanistically, ICB depends on available tumor antigen to be recognized by unleashed immune cells to mount an antitumor response and develop immune memory. Antigen density may be severely limited, or absent, after surgery, limiting efficacy. Additionally, PD-1 expression on peripheral blood mononuclear cells has been shown to dramatically diminish in patients with RCC after nephrectomy, which, for adjuvant therapy, would mean essentially losing the target before firing the first shot. ${ }^{19}$ Treating patients preoperatively or perioperatively (ie, both before and after surgery) would allow for antigen recognition when the primary tumor is intact, maximizing immunogenicity. This would also facilitate more meaningful correlative studies, as blood and tissue obtained before starting treatment could be compared with specimens collected at nephrectomy to enhance understanding of changes in the immune milieu and potentiate biomarker exploration. The downside of neoadjuvant treatment is the potential for an autoimmune toxicity that could delay, prevent, or increase the risk of curative-intent surgery.

There are other issues with planning ICB trials in early-stage disease. The selection of the comparator arm in a randomized trial (ie, placebo vs observation) is imprecise. Observation is the current standard, and thus a fair comparator, but bias in investigator assessment of both toxicity and efficacy is inherent in unblinded clinical trials. ${ }^{20}$ Placebocontrolled trials can mitigate unintended bias, but patient anxiety regarding placebo may limit accrual, and rules regarding unblinding may affect treatment options for patients who develop metastatic disease. Furthermore, as new data from the adjuvant TKI studies become available, the landscape may change, compelling design amendments that could delay and complicate trial analysis, or compel patients to forgo trials to receive sunitinib. Finally, although the side-effect profile of ICB is generally considered tolerable compared with available standard drugs, the appetite for serious AEs changes from the metastatic to curative setting. When treating an incurable disease, patients and clinicians are more willing to tolerate potentially serious and even fatal AEs. In otherwise healthy patients who may be cured with surgery alone, patients and clinicians may weigh the risks much differently, and this becomes even more relevant when studying dual ICB. Several trials are using ICB in a perioperative setting (Table 2).

\section{Non-Clear Cell RCC}

Non-clear cell histologies constitute $20 \%$ to $25 \%$ of RCCs. However, this group is heterogeneous, and although when grouped together they make up a significant proportion of RCCs, individually each subtype is relatively rare and thus difficult to study in large prospective trials. The most common nccRCC subtype is papillary, but other subtypes include chromophobe, sarcomatoid, collecting duct, medullary, and various hereditary forms. Patients with metastatic nccRCC have generally demonstrated less responsiveness to the drugs shown to be active in ccRCC. In the TKI era, although some patients with nccRCC may derive some benefit from VEGF-targeting TKIs, retrospective studies have generally suggested these agents have inferior efficacy compared with what would be expected in patients with ccRCC. ${ }^{21}$ This was also true in the previous era of immunotherapy, namely with HD IL-2. Although included in some of the larger trials of HD IL-2, patients with nccRCC rarely experienced clinical benefit. ${ }^{22-24}$ Treatment with interferon alfa has also demonstrated limited efficacy in patients with non-clear cell histologies. ${ }^{24}$ No prospective data currently exist to characterize the response of patients with nccRCC to ICB, though several case reports have been published identifying single responses across various histologies. ${ }^{25-27}$ Several ongoing studies are evaluating ICB as a single agent and in combination in patients with nccRCC.

\section{The Search for Predictive Biomarkers}

Despite the initial promise of PD-L1 expression on tumor cells as a reliable biomarker of response, in RCC this appears to have no bearing on whether patients will benefit from treatment, as evidenced by both phase II and III trials of nivolumab in mRCC. ${ }^{8,28}$ Most notably, the negative predictive value (a low or negative PD-L1 expression predicting lack of response from therapy) is poor, thus patients should not be denied ICB based on PD-L1 expres- 
Zibelman and Plimack

\section{Table 2. Perioperative Trials Including Immune Checkpoint Blockade in Renal Cell Carcinoma}

\begin{tabular}{|c|c|c|c|c|c|}
\hline $\begin{array}{l}\text { ClinicalTrials.gov } \\
\text { Identifier }\end{array}$ & Drugs & Phase & Sponsor & Design & End Points \\
\hline $\begin{array}{l}\text { Pending } \\
\text { (PROSPER RCC, } \\
\text { EA8143) }\end{array}$ & Nivolumab & III & ECOG-ACRIN & $\begin{array}{l}\text { Randomized to nivolumab } \\
\text { for } 2 \text { doses before planned } \\
\text { nephrectomy, followed by } 9 \text { mo } \\
\text { adjuvant dosing vs observation }\end{array}$ & Relapse-free survival \\
\hline $\begin{array}{l}\text { Pending } \\
\text { (IMmotion 010, } \\
\text { WO39210) }\end{array}$ & Atezolizumab & III & F. Hoffman-La Roche Ltd. & $\begin{array}{l}\text { Randomized to adjuvant } \\
\text { atezolizumab for } 1 \text { y vs placebo } \\
\text { (includes patients with R0 } \\
\text { metastasectomy) }\end{array}$ & Disease-free survival \\
\hline NCT02575222 & Nivolumab & I & $\begin{array}{l}\text { Sidney Kimmel Comprehensive } \\
\text { Cancer Center }\end{array}$ & $\begin{array}{l}3 \text { doses before planned } \\
\text { nephrectomy }\end{array}$ & Safety \\
\hline NCT02595918 & Nivolumab & Pilot & $\mathrm{NCl}$ & $\begin{array}{l}3 \text { doses before planned } \\
\text { nephrectomy }\end{array}$ & Feasibility \\
\hline NCT02212730 & Pembrolizumab & 1 & Merck Sharp \& Dohme & $\begin{array}{l}3 \text { doses before planned } \\
\text { nephrectomy }\end{array}$ & $\begin{array}{l}\text { Safety and } \\
\text { intratumoral } \\
\text { correlative analyses }\end{array}$ \\
\hline NCT02762006 & $\begin{array}{l}\text { Durvalumab } \pm \\
\text { tremelimumab }\end{array}$ & I & Case Comprehensive Cancer Center & $\begin{array}{l}\text { Varying dose and schedule } \\
\text { combinations of durvalumab } \\
\text { alone or with tremelimumab } \\
\text { before and after planned } \\
\text { nephrectomy }\end{array}$ & Safety \\
\hline
\end{tabular}

sion analysis. The reasons for PD-L1 proving to be a poor biomarker have been reviewed extensively and include variables such as tumor heterogeneity, lack of standardization of assays and what denotes a "positive" test, use of archival tissue for testing in trials, and the dynamic nature of PD-L1 expression. ${ }^{29}$ The most comprehensive assessment of complementary potential biomarkers in mRCC was performed in an exploratory study of nivolumab at varying doses in both treatment-naïve and previously treated patients. ${ }^{16}$ Although their findings demonstrated hypothesis-generating changes in tumor-infiltrating lymphocytes and upregulation of interferon $\gamma$-associated genes, no clear new biomarkers were identified. Thus, at this time, no reliable predictive model exists, and commercially available PD-L1 assays should not be used to determine who can receive ICB.

\section{Conclusions}

Immunotherapy with ICB has established itself in a prominent role in the management of $\mathrm{mRCC}$, with nivolumab leading the way and more drugs and combinations likely to follow. Despite the promise of durable responses with $\mathrm{ICB}$, the field of treatment options for mRCC has become increasingly crowded, leading to uncertainty regarding the optimal place for ICB. It clearly remains a high-priority option for most patients after failure of a first-line TKI, but as the landscape evolves, ongoing trials are likely to establish new roles, possibly in combination, and push ICB earlier in the disease lifecycle. Ample clinical research has begun aiming to define the role of ICB in localized RCC to prevent recurrence, but these trials must proceed with care because results may hinge on appropriate trial design and wholesale patient and clinician buy-in.

\section{References}

1. Everson TC. Spontaneous regression of cancer. Ann N Y Acad Sci 1964;114:721-735.

2. Hallahan J. Spontaneous remission of metastatic renal cell adenocarcinoma: a case report. J Urol 1959;81:522-525.

3. Miller HC, Woodruff MW, Gambacorta JP. Spontaneous regression of pulmonary metastases from hypernephroma. Ann Surg 1962;156:852-856.

4. Fisher RI, Rosenberg SA, Fyfe G. Long-term survival update for high-dose recombinant interleukin-2 in patients with renal cell carcinoma. Cancer J Sci Am 2000;6(Suppl 1):S55-57.

5. Fyfe G, Fisher RI, Rosenberg SA, et al. Results of treatment of 255 patients with metastatic renal cell carcinoma who received high-dose recombinant interleukin-2 therapy. J Clin Oncol 1995;13:688-696.
6. Ravaud A, Motzer RJ, Pandha HS, et al. Adjuvant sunitinib in high-risk renal-cell carcinoma after nephrectomy. N Engl J Med 2016;375:22462254.

7. Rini BI, McDermott DF, Hammers H, et al. Society for Immunotherapy of Cancer consensus statement on immunotherapy for the treatment of renal cell carcinoma. J Immunother Cancer 2016;4:81.

8. Motzer RJ, Escudier B, McDermott DF, et al. Nivolumab versus everolimus in advanced renal-cell carcinoma. N Engl J Med 2015;373:1803-1813.

9. Plimack ER, Motzer RJ, Escudier B, et al. Two-year efficacy and safety update from the phase III CheckMate 025 study of nivolumab versus everolimus in patients with advanced renal cell carcinoma (aRCC). Presented at the 2016 NCRI Cancer Conference; November 6-9, 2016; Liverpool, England. 
Immunotherapy in Renal Cell Carcinoma

10. Jonasch E, Signorovitch JE, Lin PL, et al. Treatment patterns in metastatic renal cell carcinoma: a retrospective review of medical records from US community oncology practices. Curr Med Res Opin 2014;30:2041-2050.

11. Choueiri TK, Escudier B, Powles $T$, et al. Cabozantinib versus everolimus in advanced renal-cell carcinoma. N Engl J Med 2015;373:1814-1823.

12. Motzer RJ, Hutson TE, Glen H, et al. Lenvatinib, everolimus, and the combination in patients with metastatic renal cell carcinoma: a randomised, phase 2, open-label, multicentre trial. Lancet Oncol 2015;16:1473-1482.

13. Choueiri TK, Escudier B, Powles T, et al. Cabozantinib versus everolimus in advanced renal cell carcinoma (METEOR): final results from a randomised, open-label, phase 3 trial. Lancet Oncol 2016;17:917-927.

14. Mcdermott DF, Sosman JA, Sznol M, et al. Atezolizumab, an antiprogrammed death-ligand 1 antibody, in metastatic renal cell carcinoma: long-term safety, clinical activity, and immune correlates from a phase Ia study. J Clin Oncol 2016;34:833-842.

15. Rosenberg SA, Yang JC, White DE, Steinberg SM. Durability of complete responses in patients with metastatic cancer treated with high-dose interleukin-2: identification of the antigens mediating response. Ann Surg 1998;228:307-319.

16. Choueiri TK, Fishman MN, Escudier B, et al. Immunomodulatory activity of nivolumab in metastatic renal cell carcinoma. Clin Cancer Res 2016;22:5461-5471.

17. Hammers HJ, Plimack ER, Infante JR, et al. Expanded cohort results from CheckMate 016: a phase I study of nivolumab in combination with ipilimumab in metastatic renal cell carcinoma (mRCC) [abstract]. J Clin Oncol 2015;33(Suppl):Abstract 4516

18. Haas NB, Manola J, Uzzo RG et al. Adjuvant sunitinib or sorafenib for high-risk, non-metastatic renal-cell carcinoma (ECOG-ACRIN E2805): a double-blind, placebo-controlled, randomised, phase 3 trial. Lancet 2016;387:2008-2016.

19. Macfarlane AW, Jillab M, Plimack ER, et al. PD-1 expression on peripheral blood cells increases with stage in renal cell carcinoma patients and is rapidly reduced after surgical tumor resection. Cancer Immunol Res 2014:2:320-331.
20. Moher D, Hopewell S, Schulz KF, et al. CONSORT 2010 explanation and elaboration: updated guidelines for reporting parallel group randomised trials. J Clin Epidemiol 2010;63:e1-37.

21. Kroeger N, Xie W, Lee JL, et al. Metastatic non-clear cell renal cell carcinoma treated with targeted therapy agents: characterization of survival outcome and application of the International mRCC Database Consortium criteria. Cancer 2013;119:2999-3006.

22. Mcdermott D, Ghebremichael M, Signoretti S, et al. The high-dose aldesleukin (HD IL-2)"SELECT" trial in patients with metastatic renal cell carcinoma (mRCC). J Clin Oncol 2010;28(Suppl):4514-4514.

23. Mcdermott DF, Regan MM, Clark JI, et al. Randomized phase III trial of high-dose interleukin-2 versus subcutaneous interleukin-2 and interferon in patients with metastatic renal cell carcinoma. J Clin Oncol 2005;23:133-141

24. Motzer RJ, Bacik J, Mariani T, et al. Treatment outcome and survival associated with metastatic renal cell carcinoma of non-clear-cell histology. J Clin Oncol 2002;20:2376-2381.

25. Adra N, Cheng L, Pili R. Unclassified renal-cell carcinoma with significant response to nivolumab [published online ahead of print December 29, 2016]. Clin Genitourin Cancer, doi: 10.1016/j.clgc.2016.12.019.

26. Beckermann KE, Jolly PC, Kim JY, et al. Clinical and immunologic correlates of response to PD-1 blockade in a patient with metastatic renal medullary carcinoma. J Immunother Cancer 2017;5:1.

27. Geynisman DM. Anti-programmed cell death protein 1 (PD-1) antibody nivolumab leads to a dramatic and rapid response in papillary renal cell carcinoma with sarcomatoid and rhabdoid features. Eur Urol 2015;68:912914.

28. Motzer RJ, Rini BI, Mcdermott DF, et al. Nivolumab for metastatic renal cell carcinoma: results of a randomized phase II trial. J Clin Oncol 2015;33:1430-1437.

29. Patel SP, Kurzrock R. PD-L1 expression as a predictive biomarker in cancer immunotherapy. Mol Cancer Ther 2015;14:847-856.

\section{Register now at NCCN.org/academy}

NCCN Academy for Excellence

\& Leadership in Oncology ${ }^{\mathrm{TM}}$

School of Pharmaceutical \& Biotech Business

Tuesday, July 11, 2017 • The Rittenhouse • Philadelphia, Pennsylvania

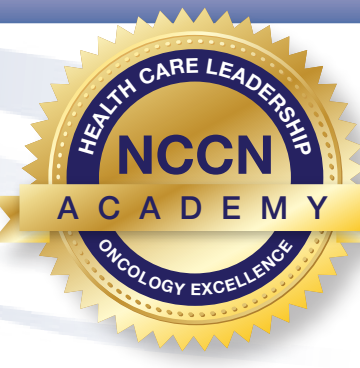

Curriculum

7:30 - 8:15 AM Breakfast and Registration

8:15 AM - 2:15 PM Module I: Ensuring Development and Access to Innovative Therapies

Module II: Meet the NCCN Guidelines Panel Experts

Module III: Patient Advocacy Update:

Policy, Programs, and Effective Collaborations

Moderator:

Clifford Goodman, PhD

Senior Vice President

The Lewin Group

\section{Closing Remarks}

2:15 PM

${ }^{*}$ Curriculum subject to change.

Visit NCCN.org/academy to enroll and to access the complete program curriculum. 